Bolshevik or unwilling to wade through its original bulk. Indeed, Haywood is along with W.E.B. Du Bois and Grace Lee Boggs one of the most important 'hands-on' guides to the U.S. Left's political and ideological turns in the century just passed. Contemporary students of African American radicalism have a special responsibility to know his name. This book will help.

Bill V. Mullen

Purdue University

\title{
Julia Creet and Andreas Kitzmann, eds. Memory and Migration: Multidisciplinary Approaches to Memory Studies (Toronto: University of Toronto Press, 2010).
}

In his 1997 critique of memory studies as an emerging field, Alon Confino warned of the narrow perspectives we develop when memory research focuses entirely on the political while neglecting the social; on representation with little attention to reception; and on asking questions about how memory manifests itself, without moving a step further and asking why. Drawing on sociologist Maurice Halbwachs' seminal work contextualizing memory within its social contexts, ${ }^{1}$ Confino argued for more nuance and complexity in studying collective memories and narratives:

To reject the separation of narratives assumes that historical actors participate in various processes at the same time, that they simultaneously represent, receive, and contest memory. To accept that none of these processes has primacy and yet to understand the meaning of memory, we need to understand all of them as intertwined-memory as a whole that is bigger than the sum of its parts. ${ }^{2}$

The challenge of how to study memory as "a whole that is bigger than the sum of its parts" still plagues the ever growing discipline of memory studies, because it necessitates being able to understand something as at once abstract and omnipresent as memory from multiple angles, through multiple frameworks. Memory is individual, social, political, and cultural at the same time. It is both internal and external. It is shaped by, and itself shapes, past, present, and future. It deals with notions of truth, subjectivity, and myth. As a result, memory studies has always been concerned with developing a multi- or inter-disciplinarydepending on who you ask — ethos and method, but just as with the wider scholarly turn towards multidisciplinary, there has been little consensus on what such a model should actually look like.

Julia Creet and Andreas Kitzmann's edited collection, Memory and Migration: Multidisciplinary Approaches to Memory Studies, rises to meet this challenge by positing movement as an ideal framework for studying memory's complexity and dynamic nature. In Creet's introduction to the volume, she provocatively 
rejects recent trends that have tied collective memory straightforwardly to place as its principal expression; rather, she sets the tone for the essays to come by arguing that, "migration rather than location is the condition of memory.... Movement always attends memory, yet we tend to take statis as its measure"(9). In his chapter, John Sundholm pushes this notion even further by stating that, "Memory is essentially a phenomenon of migration"(120). Migration here can be both physical and temporal; it is about movement, rupture and dislocation. By shifting the focus to movement, Creet argues that we can better understand how people, communities, and societies grapple with some features of memory that concern memory studies scholars more generally, such as non-linearity, fragmentation, and forgetting. It is not that we should not study the connection between memory and place, but rather that like Confino's call for understanding that "historical actors participate in various processes," Memory and Migration sees memory as grappling with various spaces, all connecting to each other in different ways, competing and contradicting each other to highlight the complexity of understanding memory, and particularly memory of violence, atrocity, and exile.

This collection takes the call to multidisciplinarity seriously, and this is its greatest strength. It is a model for what memory studies volumes should aspire to in attempting to probe mnemonic phenomena from different perspectives, and employing different theoretical frameworks. While the chapters skew towards literary studies and make heavy use of literary and critical theory, they nevertheless approach similar subjects from multiple angles. For example, the first section, "The Melancholy of No Return" examines emigration and arrival as a moment of mnemonic dislocation, and as a site ripe for study due to its instability and precariousness. It opens with Zofia Rosiska's literary essay on emigration, melancholy, and loss, which examines works such as Eva Hoffman's autobiographical writing on the Holocaust and Adam Mickiewicz's epic poem Pan Tadeusz through a psychoanalytic lens. This is followed by Srdja Pavlovic's personal essay regarding his migration from Montenegro to Canada, in which he uses Derrida among other theorists to make sense of his new hyphenated identity and the function that memory of his former life serves in constructing and deconstructing it. Veronika Zangl then examines some seminal Holocaust narratives, such as the works of Imre Kertész, Jean Améry, and Charlotte Delbo, to argue that, "One of the manifestations of troubled memories is the loss of memory" (54). This first section ends with Chowra Makaremi's troubling case study of Ghislaine K., an undocumented migrant who arrives at Roissy Airport in France from Congo-Brazzaville, and who, during her time waiting in immigration detention, struggles to negotiate between two narratives and which to tell when-one is the story her smugglers taught her, and the other is her experience as a Rwandan refugee. This chapter highlights the particular dangers of already dislocated memory interacting with bureaucratized remembering, and how these contexts operate and oppress through the exertion of mnemonic power and mis/trust; Ghislaine is eventually deported for telling the wrong story. 
The above summary of the book's first section serves as an example of how this volume itself is "bigger than the sum of its parts." The chapters are connected explicitly and thoroughly, making it easier to see the links between diverse topics and approaches. As someone whose work on troubled memory is largely based in bottom-up case studies, I read the first section with interest for the initial chapters' robust engagement with crucial memory studies theories and texts, but my own orientation made me instinctively long for these discussions to be balanced with examples of how these issues play out in people's lives. I found it deeply satisfying and ethically important that the book delivered just that wish, placing the poetic introspection of literary figures such as Charlotte Delbo alongside the contemporary bureaucratic mnemonic negotiations of Ghislaine $\mathrm{K}$. The connections between these disciplinarily different, but conceptually linked, chapters were clear; for example, in this section, Delbo's writing helps us work through memory's non-linearity, while Ghislaine K. demonstrates the disturbing consequences of non-linearity when they encounter the bureaucratization of troubled memory. These juxtapositions are present throughout the book, both within and across the sections. Section Two, which examines collective memory within diasporic communities, connects German expellees (Andreas Kitzmann), post-WWII Finns (John Sundholm), Kurds in Germany (Laurenn Guyot), and Muslim-Canadian refugees (Nergis Canefe) to make an argument for how memory is a means of working through the complex connections between here and there, and for understanding the struggle to do so. As Laurenn Guyot puts it, "Memory links the emigrant to the immigrant" (142).

Section Three then interrogates the connection between migration and sense-memory, a theme that comes together through three very different angles. Mona Lindqvist explores the psychoanalytic case of a woman's distressing memories of Uzbekistan being triggered by the smell of Hyacinths; Amira BojadzijaDan explores sense-memory in Charlotte Delbo's work to argue for viewing the body as a site of memory; and Marlene Goldman probes Margaret Atwood's novel Alias Grace for its story of migration, memory, and hysteria, arguing that it "highlight[s] an often disavowed aspect of memory studies, particularly in the context of trauma, namely, that testimony and fabrication, far from being antithetical, are in fact secret sharers of modern culture's often traumatic, gendered, and racialized experience of migration" (224). Goldman also draws parallels between her literary analysis and Makaremi's ethnographic one. The last section then turns back to space through the framework of "architectures of memory." Tomasz Mazur begins by asking, "is memory a value?" and connects Socratic writings on value to an ethics of memory (235). Luiza Nader then turns to the installation works of artist Krzysztof Wodiczko, who uses projections to highlight silenced memories on the walls of public and visible spaces. Yvonne Singer writes an autobiographical essay about seeking to reconstruct and comprehend her own family's history and experience fleeing Hungary for Canada, and how 
this obsession and feeling of fragmentation has influenced her artistic endeavours. And finally, Julia Creet discusses the dilemmas over where to donate her mother's papers, both in terms of what the location of an archive might signify about a person's sense of belonging, or lack thereof, as well as how the process of archiving personal papers places them, and thus the significance of the lives they represent, within particular frames.

This is what multidisciplinarity should look like. While each author draws on their disciplinary training and perspective, the chapters consciously cross-reference and build on each other, such that the volume has a coherent and convincing overall argument. This is not done without encountering certain quandaries, however. For example, while multiple chapters make reference to trauma as an important way that memory is disrupted and dislocated, authors define such a politically and culturally loaded term differently and to varying degrees. For example, Lindqvist approaches trauma from a clinical psychoanalytic perspective, while Sundholm compellingly argues for "cultural trauma" as a category, even as it cannot entirely mimic trauma as an internal condition within an individual. Other authors then invoke trauma without being entirely clear as to where their conception fits within the span of these two diverse possibilities. Indeed, the question of what exactly we mean by trauma highlights the continuing difficulty of trying to understand a phenomenon-memory-through a multidisciplinary lens which treats it as something profoundly internal — in the minds of individuals — and external — manifested through cultures and communitiesat the same time. The expansion of the concept of trauma beyond a particular pathology has been hugely controversial; it is surprising that this is addressed so little in a volume that covers so much conceptual and disciplinary ground. Again, this question of vocabulary and semantic precision highlights a continuing foible within memory studies as a whole that has been difficult to resolve; namely, that in trying to study the connections between individual and collective memory, we risk collapsing those levels, but even more dangerously, of treating them as though they are all equal and can be understood using the same methods. Sundholm is right to be clear that cultural trauma is not the same process or condition as individual trauma, and these distinctions need to be articulated explicitly at all times. This attention to semantic precision needs to accompany any attempt to take a term with origins in one discipline, in this case psychology, and utilize it elsewhere. As Wulf Kansteiner says, in his methodological critique of memory studies:

The fact that individual memory cannot be conceptualized and studied without recourse to its social context does not necessarily imply the reverse, that is, that collective memory can only be imagined and accessed through its manifestation in individuals. At the very least, we have to differentiate between different types of "social" memory, autobiographical memory on the one hand and collective memory on the 
other. For lack of such differentiation, many inquiries into collective memories commit a tempting yet potentially grave methodological error: they perceive and conceptualize collective memory exclusively in terms of the psychological and emotional dynamics of individual remembering. ${ }^{3}$

He then invokes the work of Iwona Irwin-Zalecka to remind us that collective memory needs to be viewed through social, cultural and political categories, and not just psychological ones. While Memory and Migration does admirable work in trying to reconcile and bring together different approaches, the varying treatments of a concept such as trauma serves as a good reminder of the challenges of multidisciplinary work: how can we learn from each other, and borrow and expand on useful concepts, without collapsing important categorical distinctions, or obscuring the specificities of particular concepts? This is not only a question for the authors of this book, but it is a question for all of us who engage with these themes.

Overall, Memory and Migration makes a convincing and thorough argument that we can best understand memory as a process and as a phenomenon by studying it in movement. Indeed, both the strengths and the challenges of this book, as described above, speak to the evolution and present limitations of memory studies as a growing field. The book, therefore, in addition to presenting compelling and original research on a host of specific topics related to its overarching theme, also makes a significant contribution to the field. It does so by successfully arguing for a reorientation of how we conceptualize memory and its connection to people and places - a reorientation that refreshingly forces us to treat memory as the complex, shifting, messy, and dynamic phenomenon that it is. ${ }^{4}$ Anton Treuer, The Assassination of Hold in the Day (St. Paul, MN: Borealis Books, 2011), 14.

\section{NOTES}

1 Anton Treuer, The Assassination of Hold in the Day (St. Paul, MN: Borealis Books, 2011), 14

2 Maurice Halbwachs, On Collective Memory, ed. and trans. Lewis A. Coser (Chicago: The University of Chicago Press, 1992).

3 Alon Confino, "Collective Memory and Cultural History: Problems of Method," The American Historical Review 102, 5 (December 1997): 1398-99.

4 Wulf Kansteiner, "Finding Meaning in Memory: A Methodological Critique of Collective Memory Studies," History and Theory 41 (May 2002): 185.

Anna Sheftel Saint Paul University 